VOL. 35 (1987) $319-320$

\title{
STABILITY IN FINITE CONTAINERS
}

\author{
BERNARD JOHN KachOYAN
}

We examine two classical problems in hydrodynamic stability theory. In particular, we study the effects of introducing finite containers into Benard convection and Dean flow (pressure driven flow between concentric cylinders). In section $I$, the linear theory of Benard convection is considered with parallel solid boundaries in one horizontal direction. These boundaries impose eight conditions on the velocity field to be found which are not present in the infinite problem. Drazin [1] has considered convective rolls parallel to the restraining walls. In our formulation, the rolls are perpendicular to these walls, that is, parallel to the short side of our box. The perturbation equations are solved by a normal-mode approach. At each value of longitudinal wavenumber $a$, an eigenrelation between the critical Rayleigh number $R$ for instability and the separation of the walls $L$ exists. This relation is solved numerically for various values of $a$. In addition a large $L$ asymptotic expansion of the Rayleigh number is derived. Comparison of the asymptotic and numerical results with those of Drazin implies that rolls perpendicular to the walls are the most unstable and are likely to occur at $a=\pi / \sqrt{ } 2$. This value of $a$ corresponds to the rolls of near-square cross-section that appear in an infinite layer.

Received 9 September 1986. Thesis submitted to University of New South Wales, February 1986. Degree approved September, 1986. Supervisor: Dr. F.J. Blennerhassett.

Copyright clearance Centre, Inc. Serial-fee code: 0004-9727/87 $\$ A 2.00+0.00$. 
In Section II, we examine cylindrical flow imposing ends on the cylinders which are assumed to move with the mean flow. This mean flow may be either Couette (Taylor vortices), Poiseuille (Dean flow) or a combination of both. Preliminary work is carried out on Taylor-Dean flow in infinite cylinders. Curves of neutral stability for this flow are found numerically using a Galerkin approach.

An asymptotic expansion of the high-wavenumber end of the neutral curves is given. The linear eigencurves in the Dean problem of critical Taylor number $T$ against cylinder height $L$ are also calculated using a Galerkin technique. It is found that solutions even and odd in the axial variable are possible and the respective $(L, T)$ curves intertwine. A weakly non-linear analysis near an intersection point $\left(L^{*}, T^{*}\right)$ of the odd and even eigensolutions gives equations to be satisfied by the amplitudes of these solutions. The coefficients of these equations are found by a direct numerical approach. Equilibrium solutions of the amplitude equations are then found and their stability examined. Unlike the Taylor vortex results of Hall [2], the properties of these solutions depend on the particular intersection point under consideration. Finally a comparison is given with previous work on the finite Dean problem.

\section{References}

[1] P.G. Drazin, "On the effects of sidewalls on Benard convection", Z. Angew. Math. Phys. 26 (1975) 239-243.

[2] P. Hall, "Centrifugal instabilities of circumferential flows in finite cylinders: nonlinear theory", Proc. Roy. Soc. London Ser. A., 372 (1980) 317-356.

Department of Applied Mathematics, University of Sydney, New South wales, 2006 Australia. 